Monográfico. Impacto de la crisis en el modelo de bienestar social.

Vulnerabilidad social y marcos alternativos

Special Issue. Impact of the crisis on the social welfare model.

Social vulnerability and alternative frameworks

Coordinado por / Guest Editor:

María Silvestre Cabrera 


\title{
Impacto de la crisis en el modelo de bienestar social. Vulnerabilidad social
}

\section{y marcos alternativos / Impact of the crisis on the social welfare model.}

\section{Social vulnerability and alternative frameworks}

\author{
María Silvestre Cabrera \\ Departamento de Trabajo Social y Sociología. Universidad de Deusto. España/Spain \\ maria.silvestre@deusto.es
}

\section{PRESENTACIÓN}

Este número monográfico de la Revista Española de Sociología (RES) recoge algunas de las principales aportaciones y conclusiones de un proyecto coordinado de investigación financiado por el Programa Estatal de Investigación, Desarrollo e Innovación Orientada a los Retos de la Sociedad del Ministerio de Economía y Competitividad (MINECORETOS) en la convocatoria de 2013-2016. El proyecto coordinado está formado por un proyecto de la Universidad de Santiago de Compostela (ARMELA DER2013-47190-C2-1-R) Crisis económica y futuro del estado de bienestar. Derechos sociales e impacto en las políticas públicas, los servicios públicos y la ciudadanía y por un proyecto del Equipo Deusto Valores Sociales ${ }^{1}$ de la Universidad de Deusto (VULNERA: DER2013-47190-C2-2-R)² Impacto de

1 El Equipo Deusto Valores Sociales es un equipo de alto rendimiento del sistema universitario vasco desde 2006. Ha obtenido en dos ocasiones consecutivas el reconocimiento como equipo de excelencia $(A)$ en las convocatorias del Departamento de Educación del Gobierno Vasco (2006-2012 y 2012-2018). La temática central del equipo es el análisis de los valores sociales, que se realiza centrado en dos grandes líneas de investigación: 1. Valores, igualdad, sociedad y democracia; 2. Valores, igualdad, bienestar y política social.

2 Este número monográfico recoge algunos de los trabajos de investigación y conclusiones del proyecto ARMELA y del proyecto VULNERA, sin embargo, las autoras de los artículos solo son una parte de los equipos de investigación y de trabajo que han participado en ambos proyectos. Procedo a mencionar a todas las personas que han participado en los proyectos y aprovecho para agradecer sus aportaciones: ARMELA (Alba Nogueira López -IP-, Alba Alonso Álvarez, M. ${ }^{a}$ Antonia Arias Martínez, Isabel la crisis en el modelo de bienestar social. Vulnerabilidad social y marcos alternativos que da título a la publicación de este número.

Estos proyectos han analizado el impacto de la crisis económica sobre los derechos sociales, los servicios públicos, el desarrollo de las políticas y el impacto en la ciudadanía desde un enfoque interdisciplinar que ha aunado disciplinas como la Sociología, el Derecho, el Trabajo Social y la Ciencia Política. La crisis económica ha operado como desencadenante de un proceso de reconfiguración del Estado de Bienestar y los cambios introducidos han tenido $-\mathrm{y}$ siguen teniendo - una notable incidencia sobre la ciudadanía, y especialmente sobre los grupos sociales más vulnerables, por lo que urge profundizar en el impacto que la crisis tiene en el modelo de bienestar social. El gran reto ha sido, por tanto, examinar la relación dinámica y compleja entre los derechos sociales, las políticas públicas y el bienestar de la ciudadanía para determinar si se garantizan de forma efectiva los derechos que conforman el marco jurídico del Estado Social español.

En el estudio realizado se ha pretendido, en primer lugar, abordar si existen límites jurídicos a los cambios 0 , en otros términos, el grado de

Diz Otero, Iñaki Lasagabaster Herrarte, Marta Lois González, Josep Ochoa Monzo e Iñigo Urrutia Libarona). VULNERA (María Silvestre -IP-, Iratxe Aristegui Fradua, Félix Arrieta Frutos, Matie Arrekoetxea Casals, Iziar Basterretxea Moreno, Usue Beloki Marañón, Irantzu Calle, Ainhoa Díez, Ester Escudero, Bakarne Etxeberria Erauskin, Ane Ferrán Zubillaga, Jone Goirigolzarri Garaizar, Edurne González Goya, Arantxa Elizondo Lopetegi, Amaia Mosteiro Pascual, Ainhoa Novo Arbona, Arantxa Rodríguez Berrio, Raquel Royo Prieto, Isabel Segú Odriozola, Emma Sobremonte Mendicuti, Ana Sofía Telletxea e Iker Usón). 
reversibilidad de los derechos sociales. También en qué medida modificaciones legislativas y competenciales en curso pueden afectar a la configuración de algunos de los pilares del Estado de Bienestar — servicios sociales, educación, igualdad, etc.- . El análisis del marco jurídico constitucional y competencial ha permitido estudiar de forma más detallada los retos que la fuerte contracción presupuestaria experimentada en los últimos años plantea a la gestión de servicios públicos. El debate sobre la universalidad de la cobertura de servicios, la cartera de prestaciones y la existencia de un núcleo indisponible en los derechos son temas cruciales en el actual contexto que enlazan también con las vías de protección de los derechos sociales y, singularmente, la protección jurisdiccional. Por otro lado, se ha estudiado la normativa de contratos del sector público en relación con los procesos de privatización de la gestión de servicios públicos y las fórmulas de colaboración público privada, por sus efectos en la garantía de la calidad de los servicios para los/ as usuarios/as. También se han Ilevado a cabo análisis de algunas de las políticas públicas en las que se sustenta el Estado de Bienestar, identificando los cambios recientes que se han producido en determinadas políticas sectoriales como consecuencia de la fuerte contracción de la intervención pública.

El proyecto VULNERA se ha centrado en el análisis del impacto que todas estas transformaciones en la normativa, los servicios públicos y las políticas — han tenido sobre las personas, y en última instancia sobre la igualdad de oportunidades.

En los últimos años se ha producido un cambio de valores que afecta a todos los ámbitos de la vida: roles de género, envejecimiento de la población, precarización del empleo, nuevas estructuras familiares y que ha tenido un correlato en la articulación de políticas públicas en materia de bienestar social. Los cambios experimentados en los modelos de convivencia familiar y en las relaciones sociales están configurando la necesidad de revisar y de innovar en los sistemas de provisión de servicios que están afectando a todos los sistemas de protección social y, como consecuencia a los procedimientos y procesos de intervención social.
Paralelamente a este cambio, se está produciendo un cambio en la percepción de la población respecto a los modelos de protección social, la prioridad asignada a lo público y a lo privado y la valoración tanto de las respuestas institucionales como del fraude social. Podemos afirmar que el cambio sociocultural está muy asociado a la "satisfacción residencial" como presupuesto clave de la que dependen otros niveles más complejos, como el profesional y el personal. No solo es importante tener en cuenta la valoración que la ciudadanía realiza de los modelos de prestación social y de los niveles de responsabilidad pública y privada a la hora de proponer modelos alternativos en la gestión del bienestar social y de los servicios sociales. También es necesario descubrir cuáles son los factores resilientes y estructurales que permiten a determinadas personas y estructuras familiares y sociales promover modelos más inclusivos.

Asimismo, en contextos de crisis resultan especialmente importante las competencias de las personas profesionales 0 voluntarias implicadas de forma directa en la interacción con las personas usuarias de Servicios Sociales o destinatarias de la intervención social, habida cuenta de la rapidez con la que determinados colectivos avanzan hacia una exclusión social grave. Si la exclusión social de forma regular es el resultado de un proceso temporal en el que interactúan entre si diferentes factores, en situaciones de crisis ese tempo se acelera de forma dramática, lo que obliga a las personas implicadas a movilizar de forma rápida sus recursos adaptativos y a gestionar las emociones que ello conlleva de manera adecuada. Ello obliga a su vez a que las personas profesionales y voluntarias movilicen y refuercen un conjunto de competencias capaces de dar respuesta a las demandas de una intervención social de alta exigencia.

En suma, la finalidad de los proyectos ARMELA y VULNERA y del número monográfico propuesto ha sido proporcionar modelos alternativos en la gestión pública y social de los servicios y prestaciones sociales desde el análisis de la valoración y percepción social, desde el descubrimiento de los factores de éxito y de fracaso de algunas políticas públicas.

El artículo "Vulnerabilidad social percibida en contexto de crisis económica" firmado por Iratxe 
Aristegui, Usue Beloki, Ainhoa Díez y María Silvestre, establece una definición de vulnerabilidad social a partir de su relación con el desempleo. La vulnerabilidad social se percibe y se vivencia en situaciones con un fuerte componente estructural y se manifiesta subjetivamente en un sentimiento de inseguridad o de indefensión. En las sociedades occidentales actuales la vulnerabilidad está estrechamente vinculada a la inestabilidad laboral, la fragilidad de las relaciones sociales y la falta de acceso a prestaciones sociales. El modelo de la vulnerabilidad explica la vivencia particular de una amenaza, como es la del desempleo, en función de los elementos amortiguadores de su impacto y de las consecuencias familiares y sociales que tiene su efectivo advenimiento. Este artículo concluye con la caracterización de tres grupos sociales vulnerables atendiendo a la situación de desempleo: "la incertidumbre eterna", "el precariado joven" y "la nueva pobreza" que se definen no solo a partir de la edad, sino con relación a la existencia de vivencias de vulnerabilidad previas y con la asunción o no de responsabilidades. Los tres tipos están atravesados por la feminización de la vulnerabilidad, puesto que las mujeres no quedan ubicadas en un único grupo de los tres señalados más arriba, sino que forman parte de las capas peor situadas 0 -precisamente más vulnerables - de cada uno de ellos, debido a cuestiones estructurales que alcanzan no sólo a aspectos materiales — como el acceso al mercado de trabajo, la precarización o la diferencia salarial—, sino también a atribuciones subjetivas de responsabilidad en el ámbito doméstico.

Este análisis de la percepción de la vulnerabilidad y de la exposición a su amenaza y a su control no puede ser ajeno a la definición de la vulnerabilidad social como una vulnerabilidad sistémica. Es decir, las personas no son vulnerables, los grupos sociales no son vulnerables, lo son determinadas situaciones y, sobre todo, lo es aquel sistema y estructura que favorece que importantes sectores de su población perciban dicha vulnerabilidad y, sobre todo, perciban la ausencia de control sobre la misma. Esta percepción de la vulnerabilidad no es ajena a las expectativas depositadas en las políticas públicas.
Dos de los artículos de este monográfico analizan la incidencia de la crisis económica en las políticas públicas; el caso de las políticas de salud sexual y reproductiva y el caso de las prestaciones económicas de la dependencia. Ambos estudios - uno más centrado en Galicia y el otro en Murcia- nos permiten afirmar que la actual crisis económica tiene un claro impacto en la planificación y ejecución de las políticas públicas y un claro impacto de género que intensifica la feminización de la vulnerabilidad social.

El artículo de Alba Alonso y Natalia Paleo, "Políticas de salud sexual y reproductiva en España: contra-movimientos y marcos interpretativos en conflicto" parte de la constatación de que el periodo de crisis económica ha representado una ventana de oportunidad para cuestionar las políticas de género. El artículo analiza las actuaciones en materia de salud sexual y reproductiva en España para explorar la naturaleza y alcance de los retrocesos experimentados e identifica la introducción de un nuevo marco interpretativo que gira en torno a la 'protección de la vida'. También estudia el papel de los actores que impulsan dicho cambio en la normativa y, a través de una investigación pormenorizada del caso gallego, explora sus consecuencias en la fase de implementación de las políticas. Con ello, contribuye a arrojar luz sobre las transformaciones en el contenido de las políticas más recientes y sobre la irrupción de nuevos actores en el sub-sistema de las políticas de género. Es posible hablar de significativos paralelismos entre las aportaciones de este artículo y las conclusiones de estudios internacionales precedentes, que invita a integrar en mayor medida el caso español en aquellas agendas de investigación comparadas en materia de retrocesos en el área de igualdad. En especial, en lo relativo al análisis del papel de los contra-movimientos, cuyo estudio es todavía incipiente en Europa.

Por su parte, el artículo de José Ángel Martínez, Lola Frutos y Juan Carlos Solano "Los usos de la prestaciones económicas de la dependencia en el municipio de Murcia. Un estudio de caso" (único artículo que no forma parte del proyecto coordinado, pero que viene a reforzar las tesis y conclusiones del mismo) se centra en el análisis de la aplicación 
de la Ley de Promoción de la Autonomía Personal y Atención a las personas en situación de Dependencia en Murcia. El artículo sostiene que la aplicación de políticas restrictivas ha transformado el sentido previsto de la ley y su gestión está ahondando en la asimetría de las relaciones de género, resultando negativa para las mujeres. La argumentación se basa en la constatación de que la prestación económica de la dependencia actúa como una renta mínima de inserción en las familias con menos recursos, con el objetivo de satisfacer sus necesidades elementales. El artículo sostiene que ante la disyuntiva de configurar un sistema de protección sustentado en los servicios o las prestaciones económicas, ha salido beneficiada esta última como consecuencia de las restricciones políticas en derechos desarrolladas en los últimos años, por la escasa oferta de servicios por parte de las administraciones públicas, por el empobrecimiento de los hogares, el escaso nivel de instrucción y el coste de oportunidad para las cuidadoras; y sobre todo por la pervivencia de un modelo de atención y protección social basado en el familismo, lo que perpetúa un modelo de desigualdad de género, al reforzar la división sexual del trabajo y las tradicionales dicotomías público/privado, productivo/reproductivo.

Dos artículos más completan este monográfico, se trata de dos investigaciones sobre la manera de garantizar derechos humanos y derechos sociales a través de las políticas públicas y de las cláusulas sociales y de la gestión directa de las políticas sociales en el caso de las políticas de desprotección familiar.

Alba Nogueira e Isabel Diz analizan el impacto que la introducción de cláusulas sociales en la contratación pública puede tener como elemento garante de los derechos sociales en el artículo "Las clausulas sociales en la contratación pública: ¿Escudo contra los recortes de derechos sociales?". El artículo tiene como objetivo avanzar en el conocimiento de las administraciones públicas como actores fundamentales para lograr la garantía de los derechos sociales y una sociedad más justa atendiendo a su actuación en la contratación pública y las cláusulas de contenido social. El artículo defiende la tesis de que uno de los ámbitos que puede contribuir a la garantía del núcleo constitucional- mente protegido de los derechos sociales es el de la contratación pública. La contratación pública estratégica guiada por criterios sociales, de género 0 ambientales puede contribuir proporcionando un cierto amparo a la garantía de derechos sociales. A pesar de los limitados márgenes para su viabilidad, que exigen afinar mucho los procesos de diseño de los contratos y pliegos de contratación, existen ya algunos ejemplos interesantes abriendo camino que favorecen la contratación con empresas que protegen el empleo de colectivos vulnerables, los salarios o realizan políticas de conciliación avanzadas. Las autoras, a partir del análisis de la contratación pública en el ámbito de la igualdad de género, concluyen que aunque está siendo todavía aplicada de manera limitada e incipiente, la potencialidad de este instrumento tanto por la amplitud del campo de intervención en las distintas dimensiones de la desigualdad que se contempla, como la efectividad práctica del instrumento a la hora de lograr objetivos concretos, la convierten en un mecanismo de la administración para garantizar el derecho a la igualdad entre hombres y mujeres.

Por su parte, el artículo "Claves del éxito o fracaso de la preservación familiar en Bizkaia: la voz de los profesionales" firmado por Arantxa Rodríguez Berrio, Amaia Mosteiro y Emma Sobremonte aporta elementos importantes para la evaluación de las políticas sociales en clave no solo de éxito sino de garante de derechos humanos. El objetivo del artículo es identificar los factores que influyen en el éxito y el fracaso del programa de preservación familiar de Bizkaia, dirigido a mejorar el funcionamiento familiar y a evitar la implementación de medidas de separación en casos de riesgo de desprotección. Los resultados obtenidos ponen de manifiesto cómo los profesionales consideran que el éxito de las intervenciones del programa de preservación familiar reside fundamentalmente en variables relacionadas con los progenitores, y no tanto con las de los niños, niñas y adolescentes, ni con las del proceso de intervención. Sus resultados nos aproximan a la comprensión de lo que podríamos denominar tentativamente un modelo de impacto sobre las variables que condicionan el éxito y fracaso de la preservación familiar y que resulta de la combinación y ponderación de los factores y variables identifica- 
das. Los resultados indican que las variables con mayor valor predictivo son las relativas a las características relacionales de la familia: competencias, motivación y voluntad de cambio de las figuras parentales y marentales junto a unas condiciones del entorno familiar protectoras. En cualquier caso, todos los programas deben de considerar los determinantes socio-estructurales que inciden en las oportunidades para modificar las situaciones. De ahí la necesidad de adoptar una mirada amplia que potencie políticas intersectoriales encaminadas a la protección infantil y que reduzcan las barreras generadoras de desigualdades sociales e inequidades. Esto significa que si los programas de preservación se centran solo en rebajar el riesgo, los esfuerzos de mejora se focalizarán fundamentalmente en las competencias parentales, pero si se quiere promover el interés superior del menor habrá que trascender el enfoque individual-familiar y adoptar una mirada crítica que, desde un enfoque de derechos humanos, considere en todos los contextos el peso de los condicionantes sociales y culturales que inciden en el bienestar infantil. De nuevo, asistimos a la posibilidad de generar cambios que protejan los derechos sociales desde la legislación y la planificación y ejecución de las políticas sociales.

Para finalizar con esta presentación, no podemos dejar de mencionar que todos los artículos muestran cómo la crisis económica ha tenido un impacto en las políticas públicas y como estas, a su vez, están teniendo un impacto de género que agranda las desigualdades entre mujeres y hombres y que nos permite hablar de feminización de la vulnerabilidad social. 
